\title{
Noninvasive Imaging of Electrophysiological Substrates in Post Myocardial Infarction
}

\author{
Linwei Wang ${ }^{1}$, Heye Zhang ${ }^{2}$, Ken C.L. Wong ${ }^{1}$, Huafeng Liu ${ }^{3}$, \\ and Pengcheng Shi ${ }^{1}$ \\ 1 Computational Biomedicine Laboratory \\ Rochester Institute of Technology, Rochester, New York, USA \\ 2 Bioengineering Institute, University of Auckland, Auckland, New Zealand \\ 3 State Key Laboratory of Modern Optical Instrumentation \\ Zhejiang University, China
}

\begin{abstract}
The presence of injured tissues after myocardial infarction (MI) creates substrates responsible for fatal arrhythmia; understanding of its arrhythmogenic mechanism requires investigation of the correlation of local abnormality between phenomenal electrical functions and inherent electrophysiological properties during normal sinus rhythm. This paper presents a physiological-model-constrained framework for imaging post-MI electrophysiological substrates from noninvasive body surface potential measurements. Using a priori knowledge of general cardiac electrical activity as constraints, it simultaneously reconstruct transmembrane potential dynamics and tissue excitability inside the 3D myocardium, with the central goal to localize and investigate the abnormality in these two different electrophysiological quantities. It is applied to four post-MI patients with quantitative validations by gold standards and notable improvements over existent results.
\end{abstract}

\section{Introduction}

The presence of injured tissues after myocardial infarction (MI) creates substrates responsible for fatal cardiac arrhythmia, such as ventricular tachycardia (VT) and fibrillation. Personalized imaging of post-MI electrophysiological substrates, particularly the correlation of local abnormality between phenomenal electrical function and inherent electrophysiological properties [1, is critical for assessing arrhythmia susceptibility of each individual.

Endocardial catheter mapping of purposely-induced VT measures electrical conduction on patient's endocardium; it guides catheter ablation with low success rate and risk of sudden death 22. Substrate voltage mapping is free from inducing VT because the electroanatomical delineation of arrhythmogenic substrate is based on the characteristics of electrograms recorded on epi- or endocardium during sinus rhythm 2]. Electrical impedance mapping reflects bulk electrical properties of heart surfaces that vary with the evolution of MI 3 . These techniques define the extent of substrate on heart surfaces, but do not depict 3D substrate structure or reveal substrate undetectable by surface recordings. Besides, their spatial resolution is limited by the number of electrodes. 
High-resolution contrast-enhanced MRI noninvasively reveals infarcted tissues with spatially complicated structures and tissue heterogeneity [1]. However, anatomical scar identified by MRI is not necessarily identical with critical electrophysiological substrates. Besides, contrast-enhanced MRI involves relatively expensive practice, use of harmful contrast agent such as gadolinium (Gd), and high false-positive identification because MI is not the only condition leading to contrast delayed Gd-enhancement 4].

Body surface potential mapping (BSPM) provides standard noninvasive observations of cardiac electrical activity with relatively simple procedure and economical equipments. Existing efforts in BSPM-driven cardiac electrophysiological reconstruction, when applied to post-MI substrate imaging, only focus on cardiac electrical functions. Electrophysiological reconstruction on heart surfaces assumes the substrate to be homogeneous and defines its extent only on heart surfaces [5]. Using a spherical infarct model, 6] estimated its location and size from BSP by deterministic optimization. The predefined infarct shape, however, does not allow flexible data-driven descriptions of intricate 3D infarct structures. We have developed a physiological-model-constrained framework that statistically combines general knowledge and patient's data for personalized imaging of volumetric TMP dynamics. It evaluates infarct solely based on abnormality of estimated TMP dynamics 7].

In this paper, we further develop our framework for noninvasive imaging of not only the phenomenal electrical function but also the inherent tissue property of the 3D myocardium for individual subjects. To obtain preliminary knowledge about local TMP abnormality, volumetric TMP dynamics is firstly estimated from BSPM data under the constraints of normally-parametrized physiological models. This initial TMP estimates is used to initialize simultaneous estimation of TMP and tissue excitability using BSPM data; abnormality in the two estimates is localized for investigation of their correlation and identification of electrophysiological substrates. Experiments are performed on four post-MI patients, where infarct location and extent are validated with the gold standard and compared to existent results on the same data sets.

\section{Methodology}

\subsection{State Space System of Cardiac Electrophysiology}

A priori physiological knowledge is used to constrain the reconstruction of subject-specific volumetric cardiac electrophysiological details. It is modeled on personalized heart-torso structures, where the volumetric myocardial TMP activity model for general spatiotemporal TMP dynamics is developed from [8]:

$$
\left\{\begin{array}{l}
\frac{\partial \mathbf{U}}{\partial t}=-\mathbf{M}^{-1} \mathbf{K} \mathbf{U}+k \mathbf{U}(\mathbf{U}-a)(1-\mathbf{U})-\mathbf{U V} \\
\frac{\partial \mathbf{V}}{\partial t}=-e(\mathbf{V}+k \mathbf{U}(\mathbf{U}-a-1))
\end{array}\right.
$$

and TMP-to-BSP model for the mapping of BSP from volumetric TMP by [7]:

$$
\Phi=\mathbf{H U}
$$




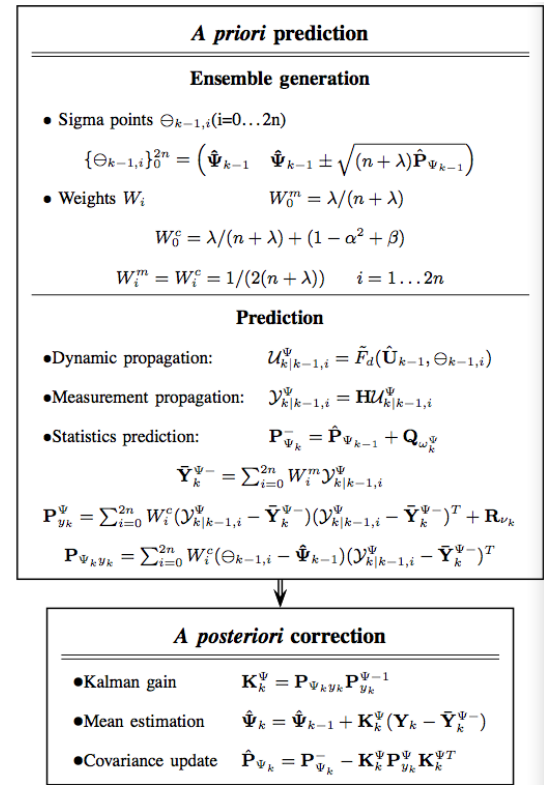

(a)

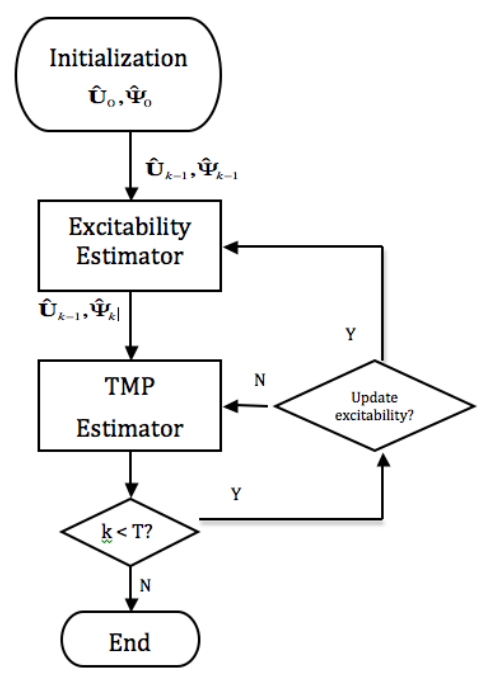

(b)

Fig. 1. (a) Flow of excitability estimation algorithm at the $k$ th iteration. $\mathbf{Q}_{\omega_{k}^{\Psi}}$ and $\mathbf{R}_{\nu_{k}}$ : pre-specified covariance matrices for $\omega_{k}^{\Psi}$ and $\nu$. (b) Dual estimator of TMP dynamics and tissue excitability. $T$ : total number of estimation steps.

where vectors $\mathbf{U}$ and $\mathbf{V}$ consist of TMP and recovery current on all meshfree points, and $\boldsymbol{\Phi}$ includes BSP from all vertices on the body surface. Matrices $\mathbf{M}$ and $\mathbf{K}$ account for intercellular electrical propagation; they encode 3D myocardial structure and its conductive anisotropy. $\mathbf{H}$ contains geometrical and conductivity information in personalized heart-torso structures. Parameters $e, k$ and $a$ determine TMP shapes, particularly, a represents myocardial tissue excitability and its increased value corresponds to reduced excitability.

To take into account modeling and data uncertainties $\left(\omega_{k}\right.$ and $\left.\nu_{k}\right)$, the physiological system is discretized into stochastic state space representation :

$$
\begin{aligned}
\mathbf{X}_{k} & =F_{d}\left(\mathbf{X}_{k-1}, \mathbf{\Psi}_{k-1}\right)+\omega_{k} \\
\mathbf{Y}_{k} & =\tilde{\mathbf{H}} \mathbf{X}_{k}+\nu_{k}
\end{aligned}
$$

where $\mathbf{X}_{k}=\left(\begin{array}{cc}\mathbf{U}_{k}^{T} & \mathbf{V}_{k}^{T}\end{array}\right)^{T}$ and $\mathbf{Y}_{k}=\boldsymbol{\Phi}_{k} . \mathbf{\Psi}$ consists of unknown parameter $a$ from all meshfree points, assumed to be spatially inhomogeneous but temporally invariant with random disturbance $\omega_{\Psi_{k}}$ :

$$
\boldsymbol{\Psi}_{k}=\boldsymbol{\Psi}_{k-1}+\omega_{\Psi_{k}}
$$

With increasing severity of injury after MI, temporal TMP shape is characterized by changes such as progressively reduced potential duration $(P D)$ and delayed 


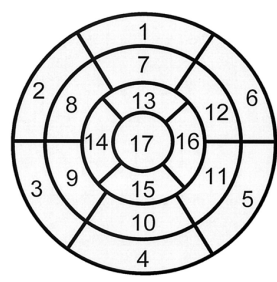

(a)

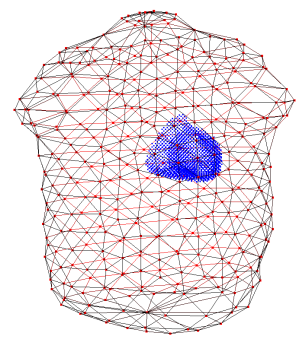

(b)

Fig. 2. (a) Standard 17-segement division of LV wall (polor map). Segments $1-6$ lie in the basal layer, $7-12$ the middle layer, 13-16 the apical layer and 17 the apex. At each layer, the segment labeling moves from anterior, septal, inferior to lateral part of the LV. (b) Personalized heart-torso model in case 2, where the ventricles are represented with 1373 meshfree points and the torso by triangulated body surface with 370 vertices.

activation time $(A T)$ until the absence of activation in necrotic tissues. This loss of myocardial viability is also reflected as decreased tissue excitability.

\subsection{Dual Estimation of TMP Dynamics and Tissue Excitability}

To obtain preliminary approximation of local TMP abnormality without personalized prior knowledge, TMP dynamics is firstly estimated from BSP under constraints of normally-parameterized models (12) as described in [7]. AT and $P D$ are calculated from TMP shapes, the order of which are then indexed by normalized $i A T$ and $i P D$ (points with earliest activation and longest TMP duration are indexed at $i A T=0$ and $i P D=0$, respectively). Differences of $i A T$ and $i P D$ between TMP estimates and simulated normal TMPs, $d A T$ and $d P D$, measure the abnormality of local TMP dynamics as $d C o m b=(d A T+d P D) / 2$. Parameter $a$ on points with distinctly high value of $d C o m b$ are assigned with large value 0.3 for impaired excitability, while the others are valued from $0.14-0.17$ according to TMP heterogeneity across the heart wall. This vector of $\boldsymbol{\Psi}_{0}$, with $\mathbf{U}_{0}$ determined by locations of normal earliest ventricular activation, is used to initialize dual $\mathbf{U}$ and $\boldsymbol{\Psi}$ estimation.

Excitability estimator is developed similarly to TMP estimator in [7. As described in Fig 1 (a), at each iteration $k$ with previous estimates $\hat{\mathbf{\Psi}}_{k-1}$ and $\hat{\mathbf{P}}_{\Psi_{k-1}}$, an ensemble set $\left\{\ominus_{k-1, i}\right\}_{i=0}^{2 n}$ is generated from $\hat{\mathbf{\Psi}}_{k-1}$ and $\hat{\mathbf{P}}_{\Psi_{k-1}}$ with $n$ as the dimension of $\hat{\boldsymbol{\Psi}}$. It is passed through the system models (1), 2) with previous TMP estimates $\hat{\mathbf{U}}_{k-1}$ to generate new ensemble sets $\left\{\mathcal{U}_{k \mid k-1, i}^{\Psi}\right\}_{i=0}^{2 n}$ and $\left\{\mathcal{Y}_{k, i}^{\Psi}\right\}_{i=0}^{2 n}$. They are used to predict statistics of the unknowns, which are then corrected to final estimates of $\hat{\boldsymbol{\Psi}}_{k}$ and $\hat{\mathbf{P}}_{\Psi_{k}}$ using KF update rules. Dual U- $\boldsymbol{\Psi}$ estimator loosely couples TMP estimator and excitability estimator sequentially in time (Fig 1 (b)): at iteration $k$ with TMP estimates $\hat{\mathbf{U}}_{k-1}$ and excitability estimates $\hat{\mathbf{\Psi}}_{k-1}$, excitability estimator utilizes $\hat{\mathbf{U}}_{k-1}$ to update $\hat{\boldsymbol{\Psi}}_{k-1}$ as described above; TMP estimator then utilizes the updated $\hat{\mathbf{\Psi}}_{k}$ for estimating $\hat{\mathbf{U}}_{k}$ and $\hat{\mathbf{P}}_{u_{k}}$ [7]. 

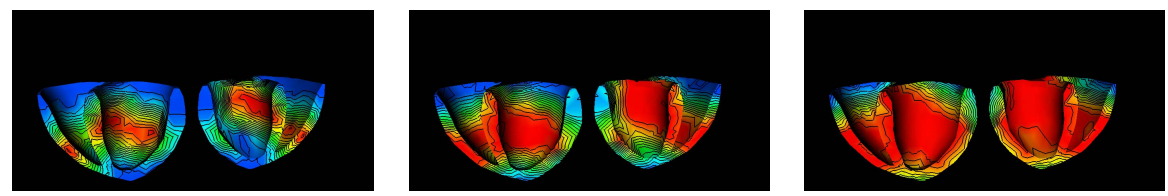

(a) Volumetric TMP dynamics simulated with normally-parametrized model.
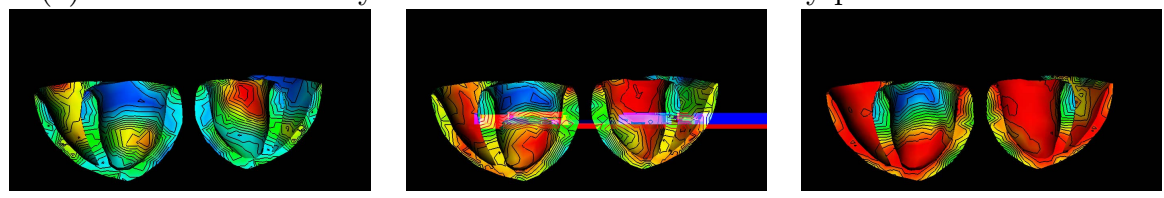

(b) Imaging of volumetric TMP dynamics using patient's BSPM data $\begin{array}{lllll}0.000 & 0.250 & 0.500 & 0.750 & 1.00\end{array}$

Fig. 3. Comparison of volumetric TMP imaging results (b) with simulated normal TMP dynamics (a) in case 2 . The color bar encodes normalized TMP values and black contours represent TMP isochrones. From left to right: $8.0 \mathrm{~ms}, 12.3 \mathrm{~ms}$ and $16.7 \mathrm{~ms}$ after the onset of ventricular activation.

\subsection{Substrate Imaging and Quantitative Evaluation}

To investigate the correlation of abnormality between TMP dynamics and tissue excitability, we measure TMP abnormality by $i A T$ and $i P D$ of the TMP estimates as $i C o m b=(i A T+i P D) / 2$, and similar thresholding method in initialization is used for distinguishing points with abnormally late $A T$ and short $P D$. Abnormal value in tissue excitability directly reflect myocardial inviability, where increasing severity of injury is represented by $a>0.25$ until the total loss of viability in necrotic tissues with $a>0.5$ [ .

For comparisons with gold standard and the existing works, we evaluate the center and extent of infarcted tissues with abnormal excitability. Using the standard 17-segment division of LV (Fig 2 (a)) [9], we identify segments containing infarct substrate and calculate its extent $(E P)$ by dividing the number of infarcted meshfree points by the total number of meshfree points. Substrate centroid $(C E)$ is localized as the segment containing the center of infarcted meshfree points weighted by excitability estimates. Segment overlap $(S O)$ with gold standard measures the percentage of correct identification.

\section{Experiments}

\subsection{Experimental Data and Data Processing}

MRI and BSP data are collected from 4 post-MI patients (case 1 - 4) [10]. Cardiac MRI of each patient contains 10 slices rom apex to base of the heart, with $8 \mathrm{~mm}$ inter-slice spacing and $1.33 \mathrm{~mm} /$ pixel in-plane resolution. After handtracing epi-/endo-cardial contours and building a smoothed mesh for the heart surfaces, we drop a could of $1000-2000$ meshfree points inside the surface mesh to represent the $3 \mathrm{D}$ heart wall. Myocardial conductive anisotropy is considered 


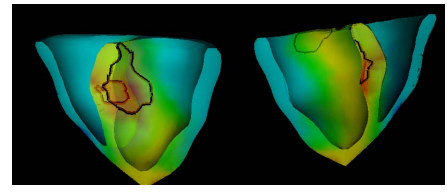

a

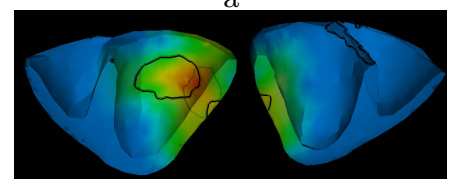

c

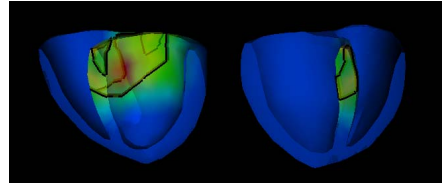

$\mathrm{b}$

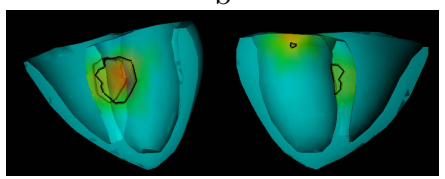

d

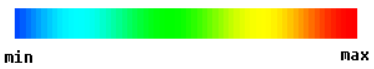

Fig. 4. Superimposed imaging of local abnormality in TMP dynamics and tissue excitability of the 3D myocardium ((a)-(d): case 1-4). Black contour encircles region of abnormal TMP functions. Color encodes tissue excitability; higher value represents lower excitability. Half-transparent visualization is used for better observationl.

by mapping volumetric fiber structures from the mathematical fibrous model in [11. The torso is assumed to be an isotropic and homogeneous volume conductor, described by triangulated body surface with 370 apexes and obtained by deforming a reference torso model to match patient's MRI data 12. Fig 2 (b) illustrates the personalized heart-torso model in case 2 .

BSP is recorded by 123 electrodes with known anatomical locations and interpolated to 370 apexes on the body surface. Complete BSP sequences consist of a single averaged PQRST complex sampled at $2 \mathrm{k} \mathrm{Hz}$, out of which QRST interval for ventricular electrical activity is selected as framework inputs. It is interpolated in time and scaled in magnitude to adapt to the discretization step and normalized TMP value of the TMP activity model (1). There are no available gold standards of volumetric TMP activity or tissue excitability. Instead, after examining Gd-enhanced cardiac MRI, cardiologists provid us infarct center $(C E)$, extent $(E P)$ and affected segments for 4 cases (Table 10) 10.

\subsection{Results}

In volumetric TMP imaging results of case 2 (Fig 3 (b)), septal-inferior part of basal-middle LV exhibits distinct conduction delay compared to normal TMP dynamics simulated in the patient's heart (Fig 3 (a)). Fig 4 (b) illustrates the superimposed TMP and excitability abnormality, where the region of abnormal TMP functions is encircled with black contours and the color encodes tissue excitability. Fig 4 (a), (c) and (d) list results for case 1, 3 and 4, respectively: infarct collects in septal-anterior part of basal LV and septal part of middle LV in case 1 , the inferior part of basal-middle LV and lateral part of middle-apical LV in case 3, and in anterior-basal, septal-middle LV in case 4 . These results are in accordance with gold standards. Similar to the observations in [1, while 
Table 1. Comparison of MI evaluation results with gold standards (Ref). Definition of the evaluation parameters is explained in the text.

\begin{tabular}{|c|c|c|c|c|c|c|c|c|}
\hline \hline & \multicolumn{2}{|c|}{ case 1 } & \multicolumn{2}{c|}{ case 2} & \multicolumn{2}{c|}{ case 3 } & \multicolumn{2}{c|}{ case 4 } \\
\hline & Ref & Results & Ref & Results & Ref & Results & Ref & Results \\
\hline$E P$ & $31 \%$ & $17 \%$ & $30 \%$ & $20 \%$ & $52 \%$ & $30 \%$ & $15 \%$ & $11 \%$ \\
\hline$C E$ & 8 & 9 & $3 / 4 / 9 / 10$ & 9 & $10 / 11$ & 11 & 15 & 9 \\
\hline$S O$ & N/A & $97 \%$ & N/A & $100 \%$ & N/A & $100 \%$ & N/A & $100 \%$ \\
\hline segments & $1-3,8-9$ & $1-3,8-9$ & $3,4,9,10$ & $3,4,9,10$ & $3-5,9-12$ & $5,10-12$ & $1,9-11$ & 1,9 \\
& $13-15$ & $13-15,17$ & & & $15-16$ & 16 & 15,17 & \\
\hline \hline
\end{tabular}

Table 2. Comparison of MI evaluation with existent results. EPD and $C E D$ are the difference between those as estimated and as determined from gold standards.

\begin{tabular}{|c|c|c|c|c|c|c|c|c|c|}
\hline \hline & \multicolumn{2}{|c|}{ Current results } & Previous results & \multicolumn{2}{c|}{ Mneimneh } & \multicolumn{2}{c|}{ Dawoud } & \multicolumn{2}{c|}{ Farina } \\
\hline & case 3 & case 4 & case 3 & case 3 & case 4 & case 3 & case 4 & case 3 & case 4 \\
\hline$E P D$ & $22 \%$ & $4 \%$ & $24 \%$ & $25 \%$ & $2 \%$ & $17 \%$ & $26 \%$ & $43 \%$ & $14 \%$ \\
\hline$C E D$ & 0 & 1 & 0 & 0 & 1 & 1 & 2 & 1 & 1 \\
\hline$S O$ & $100 \%$ & $100 \%$ & $90 \%$ & $90 \%$ & $25 \%$ & $56 \%$ & $30 \%$ & $40 \%$ & $17 \%$ \\
\hline \hline
\end{tabular}

abnormal electrical functions occurs within infarct zone, border zone exhibits normal electrical functions. The extent of injured tissues, therefore, is not the only measure for myocardial arrhythmia susceptibility. It is the distribution of tissue heterogeneity and the direction of electrical activation that work in concert to provoke arrhythmia.

Table 1 compares gold standards with our quantitative evaluation of infarct based on tissue excitability. In case 1, we correctly identify all infarcted segments but overestimate the infarct extent down to the apex (false positive at segment 17) and localize $C E 1$ segment away from gold standards. In case 2, we precisely identify the 4 infarcted segments and correctly localize $C E$. In case 3 , we correctly localizes $C E$ and highlights 5 out of 9 infarcted segments, underestimating the infarct extent in inferior LV. Among the two separate infarct mass in case 4 , our results only identify the anterior-basal part and a small portion in inferior-middle LV. In summary, the presented framework provides close localization of $C E$ and identifies substrate with high precision $S O$ (low false positive identifications). Because calculation of EP may differ between gold standards and our approach, the reason for its discrepancies remains unclear.

Table 2 compares the current results with our previous MI evaluation which solely depends on volumetric TMP estimates [7], as well as existent results of case 3 and 4 obtained using case 1 and 2 for training. In brief, Dawoud et al solved the problem by epicardial potential imaging from BSP [5], Farina et al estimated the site and size of spherical infarct models [6], and Mneimneh et al produced the best results using simple ECG signal analysis 13 . In both cases, our results are comparable to the best results and substantially improved over the other two reconstruction results. Note that our framework has not required any training. In 
the future, proper training is a possible strategy for improving its performance. Compared to our previous work [7, simultaneous estimation of volumetric TMP dynamics and tissue excitability improves the accuracy of infarct identification (improved $E P$ and $S O$ ).

\section{Conclusions}

Our framework enables investigation of the correlation between electrical functions and tissue property inside specific subject's heart; it helps assessing the arrhythmia susceptibility of individual subjects. Future works will analyze the pathophysiological implications of the current substrate imaging results and the extension to a wider category of pathologies. Since cardiac electrical conduction depends on both the active membrane properties of cardiac cells (e.g., excitability) and passive properties determined by myocardial architectural features (e.g., conductivity), future studies will investigate whether it is possible, and if so, how to separately estimate post-MI excitability and conductivity using BSPM data.

\section{References}

1. Ashikaga, H., Sasano, T., Dong, J., et al.: Magnetic resonance-based anatomical analysis of scar-related ventricular tachycardia. implications for catheter ablation. Circ. 101, 939-947 (2007)

2. Volkmer, M., Ouyang, F., Deger, F., et al.: Substrate mapping vs. tachycardia mapping using carto in patients with coronary artery disease and ventricular tachycardia: impact on outcome of catheter ablation. Europace 8, 968-976 (2006)

3. Fallert, M.A., Mirotznik, M.S., Downing, S.W., et al.: Myocardial electrical impedance mapping of ischemic sheep hearts and healing aneurysms. Circ. 87, 199-207 (1993)

4. Arai, A.E.: False positive or true positive troponin in patients presenting with chest pan but normal coronary arteries: Lessons from cardiac MRI. European Heart Journal 28, 1175-1177 (2007)

5. Dawoud, F.D.: Using inverse electrocardiography to image myocardial infarction. In: Proc. Comput. Cardiol. (2007)

6. Farina, D.: Model-based approach to the localization of infarction. In: Proc. Comput. Cardiol. (2007)

7. Wang, L., Wong, K.C.L., Zhang, H., Shi, P.: Noninvasive functional imaging of volumetric cardiac electrical activity: A human study on myocardial infarction. In: Metaxas, D., Axel, L., Fichtinger, G., Székely, G. (eds.) MICCAI 2008, Part I. LNCS, vol. 5241, pp. 1042-1050. Springer, Heidelberg (2008)

8. Aliev, R.R., Panfilov, A.V.: A simple two-variable model of cardiac excitation. Chaos, Solitions \& Fractals 7(3), 293-301 (1996)

9. Cerqueira, M.D., Weissman, N.J., Dilsizian, V., et al.: Standardized myocardial segmentation and nomenclature for tomographic imaging of the heart. Circ. 105, 539-542 (2002) 
10. Goldberger, A.L., Amaral, L.A.N., Glass, L., et al.: Physiobank, physiotoolkit, and physionet components of a new research resource for complex physiological signals. Cric. 101, e215-e220 (2000)

11. Nash, M.: Mechanics and Material Properties of the Heart using an Anatomically Accurate Mathematical Model. PhD thesis, Univ. of Auckland, New Zealand

12. Cheng, L.: Non-invasive Electrical Imaging of the Heartl. PhD thesis, Univ. of Auckland, New Zealand (2001)

13. Mneimneh, M.A., Povinelli, R.J.: Rps/gmm approach toward the localization of myocardial infarction. In: Proc. Comput. Cardiol. (2007) 